\title{
Durable Complete Response of Refractory, Progressing Metastatic Melanoma After Treatment with a Patient-Specific Vaccine
}

\author{
Robert O. Dillman, ${ }^{1-3}$ Andreea A. Nanci, ${ }^{1,3}$ Scott T. Williams, ${ }^{4}$ Richard B. Kim, ${ }^{5}$ Russell L. Hafer, ${ }^{6}$ \\ Colleen L. Coleman, ${ }^{7}$ Peter C. Wang, ${ }^{7}$ Christopher M. Duma, ${ }^{5}$ Peter V. Chen, ${ }^{6}$ \\ Senthamil R. Selvan, ${ }^{2}$ Andrew N. Cornforth, ${ }^{2}$ and Carol DePriest ${ }^{8}$
}

\begin{abstract}
A patient with metastatic melanoma who experienced a durable complete response after treatment with a patient-specific vaccine has been described in this article. This 59-year-old woman presented with cervical spine metastases and, within the year, had experienced local disease progression and, despite various therapies, metastases to the axilla, rectum, gall bladder, and multiple soft-tissue sites. She had previously received radiation therapy, combination chemotherapy, interleukin-2 plus interferon biotherapy, and gamma knife radiosurgery, and undergone multiple surgical resections. At the time vaccine therapy was initiated, she had multiple, new, measurable, soft-tissue metastases that were increasing in size. She was treated with a vaccine consisting of autologous dendritic cells incubated with irradiated tumor cells from an autologous tumor cell line and suspended in granulocyte-macrophage colony stimulating factor (GM-CSF), with subcutaneous injections once a week for 3 weeks and monthly for 5 months. There was evidence of disease regression by the completion of therapy. A few months later a complete response was documented by radiologic scans, and subsequently reconfirmed at 6-month intervals. She remains in complete remission $>2.5$ years after starting the vaccine, and $>2$ years after completing the vaccine, and survives $>4$ years after her initial presentation with bone, bowel, and lymph node metastases. This is the first time she has been in a complete remission since her initial diagnosis. Patient-specific vaccines can sometimes induce durable complete regression of progressing soft-tissue melanoma metastases.
\end{abstract}

Key words: autologous tumor cells, dendritic cells, metastatic melanoma, vaccine

\section{Introduction}

T he safety and therapeutic potential of patient-specific autologous vaccines derived from short-term autologous tumor cell lines with most of the experience in patients with metastatic melanoma have been investigated. ${ }^{1-3}$ In 74 patients with metastatic melanoma, who were treated with an irradiated tumor cell vaccine derived from an autologous tumor cell line, a median survival of 20.5 months was observed, and at a median follow-up of 6 years, the 5-year survival was $29 \%{ }^{2}$ In a subsequent trial, in 54 patients with metastatic melanoma treated with a patient-specific vaccine consisting of autologous dendritic cells loaded with antigen from the patient's autologous tumor cell line, the median survival was $>5$ years with a $54 \% 5$-year survival for all-cause mortality at a median follow-up of 4.5 years. ${ }^{4}$ The patients enrolled in these trials included those with measurable disease, nonmeasurable disease, and no evidence of disease. Impressive

\footnotetext{
${ }^{1}$ Hoag Cancer Center, Newport Beach, California.

${ }^{2}$ Hoag Cell Biology Laboratory, Hoag Cancer Center, Hoag Hospital, Newport Beach, California.

Departments of ${ }^{3}$ Medical Oncology, ${ }^{4}$ Radiology, ${ }^{5}$ Neurosurgery, ${ }^{6}$ Radiation Oncology, and ${ }^{7}$ General Surgery, Hoag Cancer Center, Hoag Hospital, Newport Beach, California.

${ }^{8}$ Clinical Trials Office, Hoag Cancer Center, Hoag Hospital, Newport Beach, California.
}

Address correspondence to: Robert O. Dillman; Hoag Cancer Center; One Hoag Drive, Bldg 41, Newport Beach, CA 92658 E-mail: rdillman@hoaghospital.org 
A
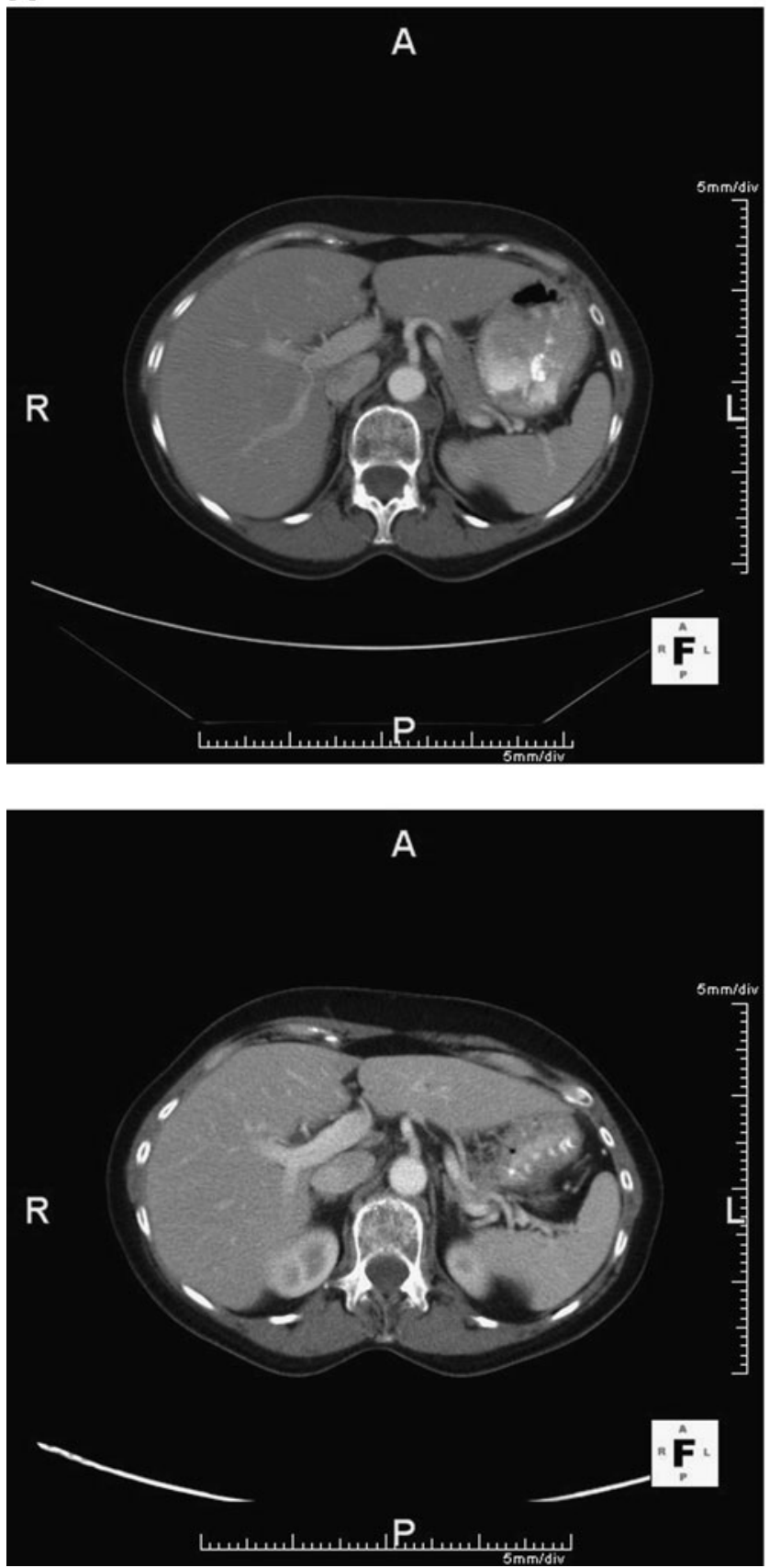

B
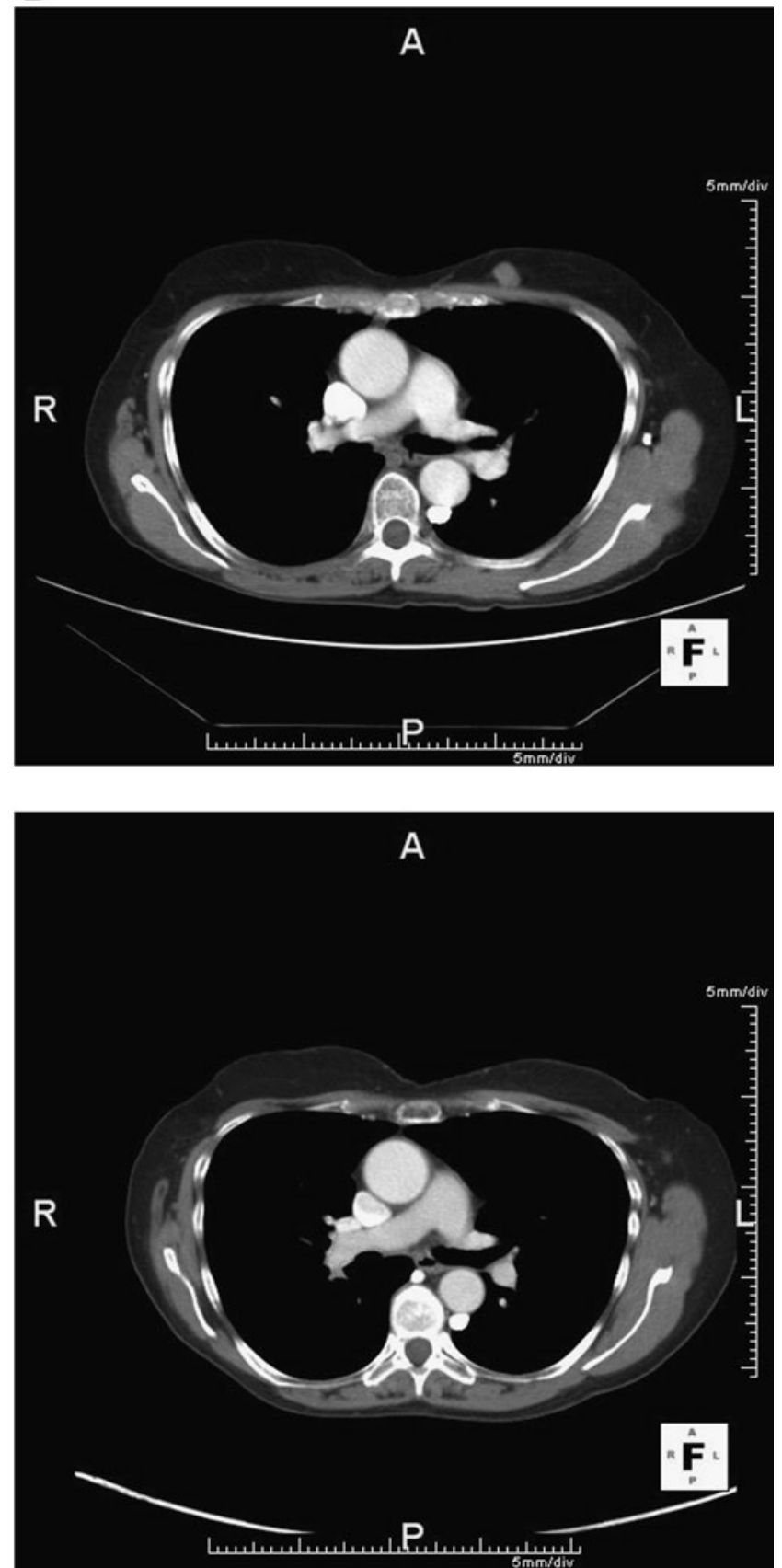

FIG. 1. Resolution, following patient-specific dendritic cell vaccine, of five different soft-tissue metastases that had been progressing in size: (A) retrocrural, (B) left breast, (C) anterior abdominal wall, (D) left lateral abdominal wall, and (E) left gluteus, all before (top) and 6 months after (bottom) completing patient-specific melanoma vaccine.

long-term progression-free survival was documented for many patients, ${ }^{3,4}$ but there were no radiographically documented objective responses of measurable melanoma. Described in this report is a patient with widespread metastatic melanoma, who already had been treated with a variety of systemic and local modalities, and subsequently experienced progressive soft-tissue disease in multiple sites that completely regressed in association with the vaccine therapy. She has remained progression free for $>2.5$ years since starting her patient-specific vaccine therapy, and continues in complete remission as of August 2010.

\section{Case Report}

In June 2006 a 59-year-old white woman presented with progressively worsening right shoulder pain, numbness, and right upper extremity weakness that progressed to severe paresis of the right arm and hand. A cervical spine magnetic resonance imaging revealed a soft-tissue mass in the right epidural space, extending from C3-C4 to T1-T2 vertebral area. She underwent a decompression laminectomy and surgical debulking of intra-spinal and extra-dural tumor. Pathology revealed poorly differentiated metastatic mela- 

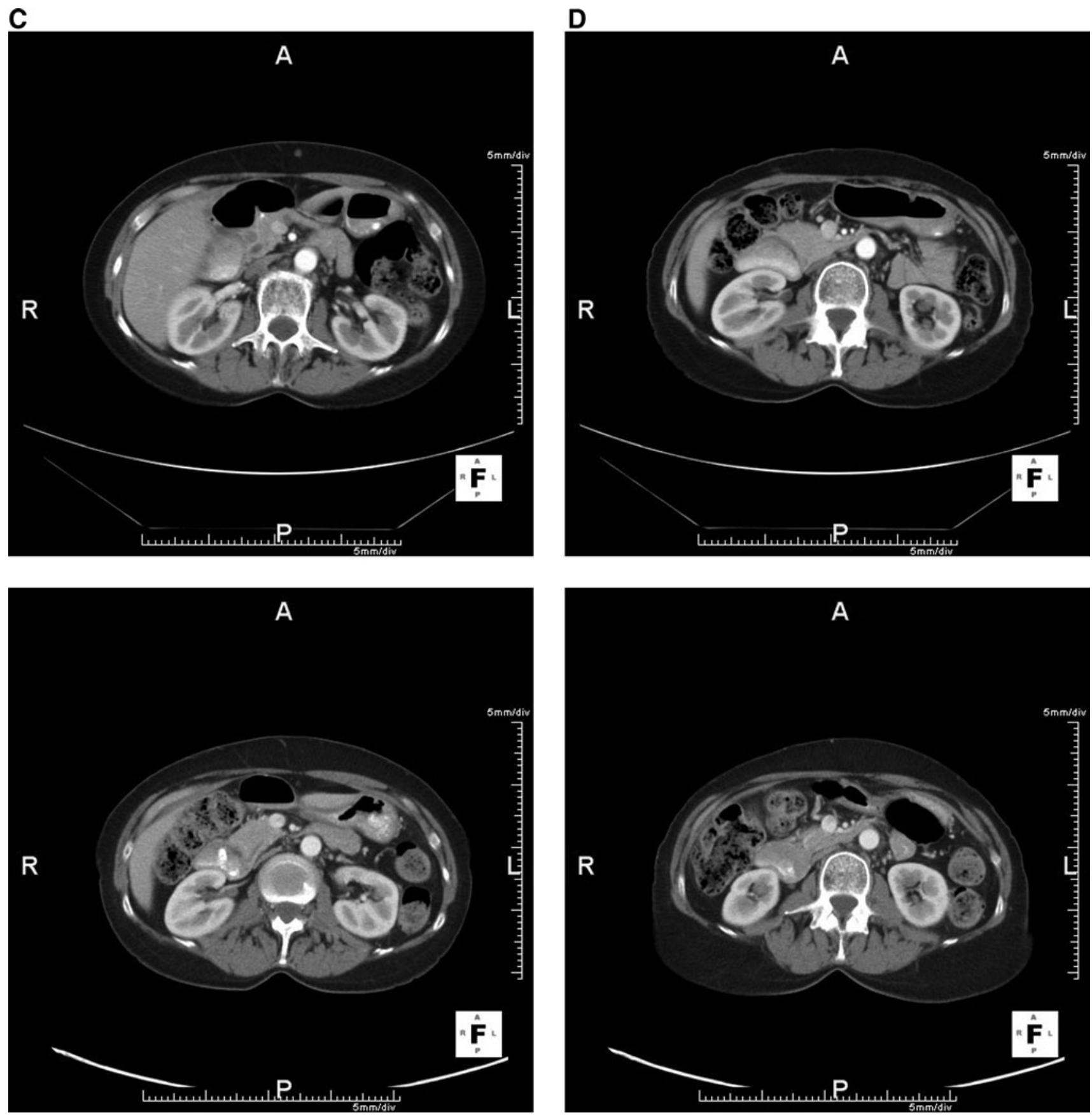

FIG. 1. (Continued).

noma expressing tumor markers S100, MART-1, and HMB45. Positron emission tomography (PET)/computed tomography scans identified additional sites of disease in the right middle lobe of the lung, the rectum, and sigmoid colon. Adjunctive radiation therapy was given to the cervical spine, consisting of 6000 cGy in 30 fractions over 53 days (cord dose restricted).

Just before starting radiation therapy, polypoid rectal and sigmoid lesions were resected during colonoscopy and confirmed to be melanoma. After completion of radiation therapy, a new right axillary metastasis was excised and efforts to establish a cell line were initiated. Because of progression of other new sites of soft-tissue metastatic disease, she started treatment with carboplatin, paclitaxel, and sorfenib, but this was associated with a minimal response. In February 2007 she was found to have two brain metastases, one in the left occipital lobe and one in the right parietal lobe, which were treated with gamma knife stereotactic radiosurgery.

Because of further disease progression, she was treated with 4 monthly cycles of a hybrid bolus/continuous infusion interleukin-2 and interferon regimen. ${ }^{5}$ In July 2007 she underwent urgent cholecystectomy, and the gall bladder was found to be extensively involved with metastatic melanoma. 
E
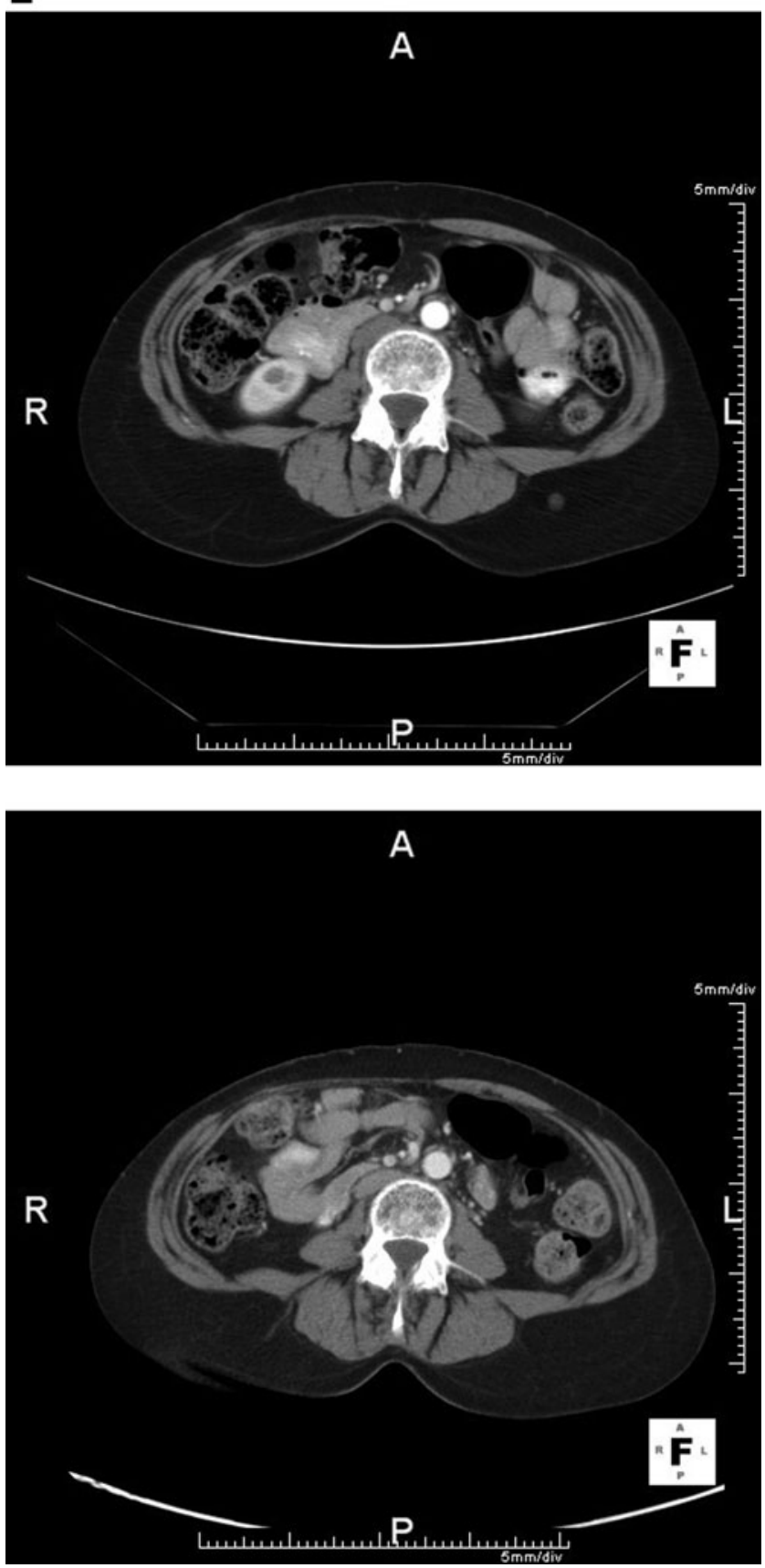

FIG. 1. (Continued).

This tissue was used to establish a second cell line. By September 2007 her performance status had deteriorated to the Eastern Cooperative Oncology Group (ECOG) 1, and she had new progressing soft-tissue metastases in several locations, including buttock, breast, abdominal wall, and retrocrural space (Fig. 1).

In October 2007 she enrolled in a randomized phase-II trial (NCT00436930) in which patients receive either the irradiated tumor cell or dendritic cell vaccine products that had previously been described. ${ }^{1-4}$ She was randomized to the dendritic cell vaccine. She underwent a leukopheresis from which dendritic cells were generated, and these were com- bined with her irradiated tumor cells from her 2 cell lines. Per protocol she received 3 weekly and then 6 monthly subcutaneous injections of her vaccine product administered in $500 \mu \mathrm{g}$ of GM-CSF over 6 months from November 7, 2007, to April 23, 2008. Three months into treatment the lesions were stable; by the end of treatment, they were all smaller and continued to regress. She received no additional treatment for melanoma after initiating the vaccine therapy. By January 2009 , scans confirmed a complete remission with continued control of all previous sites of disease (Fig. 1). Follow-up scans have continued to confirm complete remission, with the most recent completed in July 2010, > 2.5 years from the start of vaccine therapy.

\section{Discussion}

Complete remissions in response to dendritic cell-based melanoma vaccine therapy are rare events. ${ }^{6,7}$ Although patient-specific vaccines can be associated with long-term disease control and survival despite previous recurrences of widespread metastatic melanoma, ${ }^{2-4}$ this is the first complete regression with a dendritic cell-based patient-specific vaccine therapy in patients with measurable metastatic melanoma. In the most recent trial, no objective responses were recorded in 15 patients with measurable metastatic disease at the time vaccine treatment was initiated. ${ }^{4}$ In an earlier trial, 3 of 35 patients with measurable disease were reported to have had an objective response following an autologous irradiated tumor cell vaccine, but 2 of those responses were based only on physical examination of a few lesions rather than extensive radiologic staging with PET and computed tomography scans. $^{2}$

This patient had undergone a variety of other aggressive treatments that repeatedly reduced her tumor burden, and undoubtedly contributed to her survival. However, before initiating the vaccine therapy, her longest duration of progression-free survival had been no more than 5 months, and she had never been considered to be in a complete remission. The only sites of disease at the time vaccine treatment was initiated were located in soft tissues, and all represented new sites of disease, rather than older sites that had progressed during previous therapy.

\section{Conclusions}

Patient-specific vaccines can sometimes induce durable complete regression of progressing soft-tissue melanoma metastases.

\section{Disclosure Statement}

No competing financial interests exist.

\section{References}

1. Dillman RO, Beutel LD, Barth NM, et al. Irradiated cells from autologous tumor cell lines as patient-specific vaccine therapy in 125 patients with metastatic cancer: Induction of delayed-type hypersensitivity to autologous tumor is associated with improved survival. Cancer Biother Radiopharm 2002;17:51.

2. Dillman RO, DePriest $C$, DeLeon $C$, et al. Patient-specific vaccines derived from autologous tumor cell lines as active 
specific immunotherapy: Results of exploratory phase I/II trials in patients with metastatic melanoma. Cancer Biother Radiopharm 2007;22:309.

3. Dillman RO, Selvan SR, Schlitz PM. Patient-specific dendritic cell vaccines for metastatic melanoma. N Engl J Med 2006; 355:1179.

4. Dillman RO, Selvan SR, Schiltz PM, et al. Phase II trial of dendritic cells loaded with antigens from self-renewing, proliferating autologous tumor cells as patient-specific antitumor vaccines in patients with metastatic melanoma: Final Report. Cancer Biother Radiopharm 2009;24:311.
5. Dillman RO, Wiemann MC, VanderMolen LA, et al. Hybrid high-dose bolus/continuous infusion interleukin-2 in patients with metastatic melanoma: A phase II trial of the Cancer Biotherapy Research Group (formerly the National Biotherapy Study Group). Cancer Biother Radiopharm 1997;12:249.

6. O'Rourke MG, Johnson Mk, Lanagan CM, et al. Dendritic cell immunotherapy for stage IV melanoma. Melanoma Res 2007; 17:316.

7. Eubel J, Enk A. Dendritic cell vaccination as a treatment modality for melanoma. Expert Rev Anticancer Ther 2009; 9:1631. 
